(C) 2018 IEEE. Personal use of this material is permitted. Permission from IEEE must be obtained for all other uses, in any current or future media, including reprinting/republishing this material for advertising or promotional purposes, creating new collective works, for resale or redistribution to servers or lists, or reuse of any copyrighted component of this work in other works. doi: 10.1109/MPEL.2018.2850698

Publisher version: https://ieeexplore.ieee.org/document/8458285

\title{
Enabling high penetration of power electronics in the electric grid through a Global Real-Time Super Lab
}

\author{
Antonello Monti*, Marija Stevic*, Steffen Vogel*, Rik W. De Doncker*,

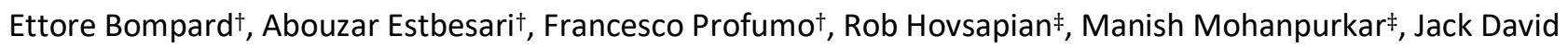

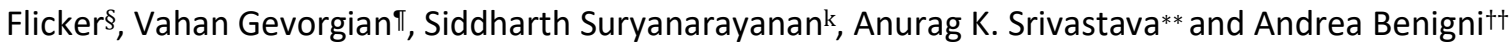 \\ *RWTH Aachen University, Aachen, Germany \\ Email: \{amonti,mstevic,stvogel,dedoncker\}@eonerc.rwth-aachen.de \\ †Politecnico di Torino, Turin, Italy \\ Email: \{ettore.bompard,abouzar.estebsari,francesco.profumo\}@polito.it \\ \#ldaho National Laboratory, Idaho Falls, USA \\ Email: \{manish.mohanpurkar,rob.hovsapian\}@inl.gov \\ §Sandia National Laboratory, Idaho Falls, ID, USA \\ Email: jdflick@sandia.gov \\ INational Renewable Energy Laboratory, Golden, CO, USA \\ Email:vahan.gevorgian@nrel.gov \\ kDept. of ECE, Colorado State University, Fort Collins, CO, USA Email: \\ sid.suryanarayanan@colostate.edu \\ **Dept. of EECS, Washington State University, Pullman, WA, USA Email: \\ anurag.k.srivastava@wsu.edu \\ t+University of South Carolina, Columbia, SC, USA \\ Email: benignia@cec.sc.edu
}

Abstract

Global Real-Time Super Laboratory (Global RT-Super Lab) represents a vendor-neutral distributed platform established based on virtual interconnection of Digital Real-Time Simulators (DRTS) and Hardware-In-the-Loop (HIL) setups hosted at eight geographically distributed laboratories located in the USA and Europe. This article describes the efforts towards the realization of this large-scale virtual infrastructure and demonstration of the multi-lab setup for simulation and testing of next generation global power grids.

\section{INTRODUCTION}

The electric grid is changing. More in particular, power electronics is significantly transforming the power system all around the world. This change is driven by the progressive installation of Distributed Energy Resources (DER) that are typically based on a power electronics interface. This transformation is creating a completely new power-electronic driven low-inertia grid. As described in [1], this future is also closer than what we can think. Even today operations of portion of grids with only power electronics-driven sources are possible. What does that mean? In a nutshell, we are transforming an electromechanical system to an electronic system. At a first glance this is perceived as a challenge. The electromechanical system presents a dynamic behavior which is fully predictable and it can be well-represented using the 2 nd Law of Newton. The consequence is that, without any control action, the system will have an intrinsic tendency to move to a new steady state operating point. This feature has been used in the classical grid to achieve system level automation without requiring high performance control and ultrafast control reaction. The first line of reaction of the system is programmed in the physics. With power electronics this feature is gone: the response depends on the control. While this is perceived as a challenge, it can actually be seen as an opportunity. A new world is open for power electronics and power system engineer to design the grid of the future. In this sense many ideas are emerging. Converters connected to the grid can present different behavior [2] depending on their control architecture. Particularly interesting is the emerging need of converters that are able to perform the so-called "grid-forming" operation. These 
converters are really the key players in supporting frequency control in the future. Said that, a lot of degree of freedom are still open for the control designer to define how this support can be delivered [3] [4]. At the same time, thanks to Ultra-High-Voltage DC application, it is also possible for the first time to link continents such as Europe and America. Plans are on the way to build a global grid to better support a futuristic scenario based on a very high penetration of renewable energy sources. It then becomes natural to ask, how will such a global grid operate? Thinking in terms of a global grid interconnecting local power electronics driven grids opens completely new theoretical questions and scenarios. Such questions regarding the dynamic operation of the system but also new issues related to stability and interoperability at an intercontinental scale. However, testing new ideas on a real grid is not an easy task. This is the reason why real-time simulation is earning so much momentum in the power community. Computational capability, limits in the data processing and also availability of real-life data limits the possibilities to perform meaningful studies in a single laboratory. For this reason, and to encourage new way of sharing knowledge among the scientists, the idea of performing distributed real-time simulation is emerging [5]. In the following, an ambition vision of a network of laboratory across two continents is introduced together with the first experimental acquired knowledge.
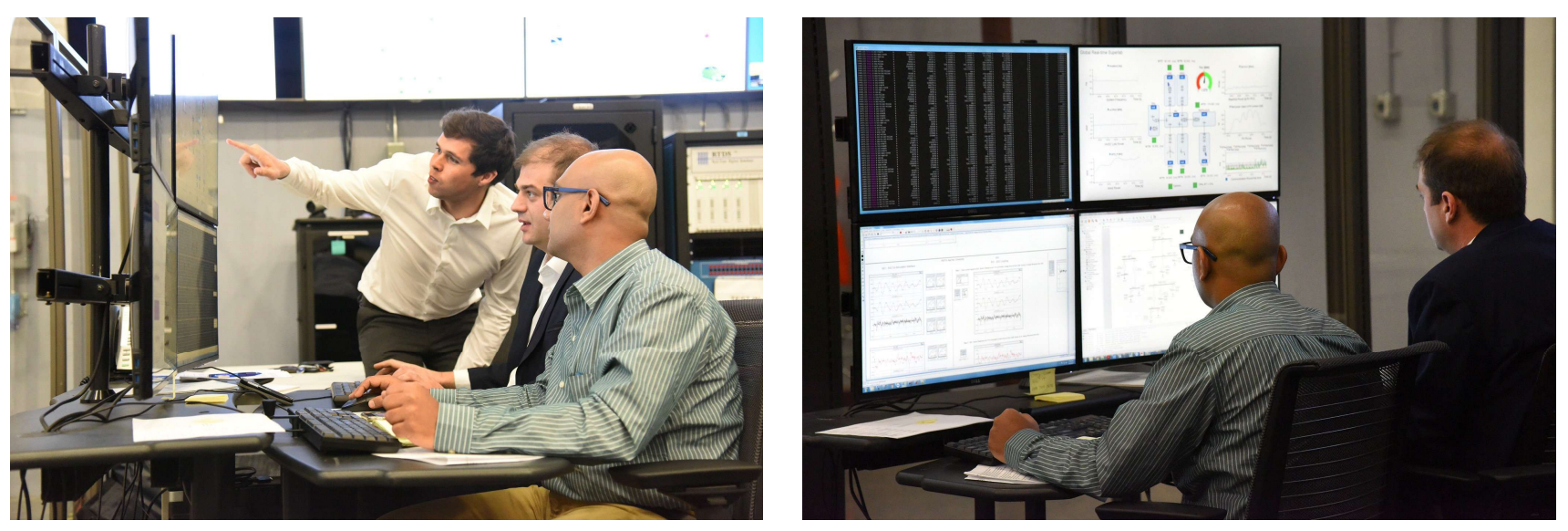

Fig. 1: Control Center at INL during RT-Super Lab demonstration.

\section{GeOgraphically Distributed Real-Time Simulation}

The paradigm shift of electric grids constituents in the form of power electronics creates two major challenges. First, largescale and multi-domain simulation and testing resources are necessary for the design and assessment of electric grids. Second, a harmonized transition towards next-generation electric grids requires a holistic approach for evaluation of synergies between newer concepts and identification of potential unpredictable interactions. To address these challenges, research facilities utilize multiple units of Digital Real-Time Simulators (DRTS) operating in parallel [6] and deploy comprehensive test benches. However, it is cost prohibitive and hardly feasible to establish a large-scale and holistic simulation and testing infrastructure at an individual facility. To this end, the concept of virtual interconnection of laboratories in real time is proposed.

Real-time simulation resources, Power Hardware-In-the-Loop (PHIL) setups, novel test benches and hybrid co-simulation frameworks are interconnected into comprehensive research infrastructure that allows the share of resources and enables largescale and multi-domain experiments. Virtually interconnected infrastructures serve as a flexible framework for collaboration with realization of joint experiments and studies based on indirect data sharing without presenting confidential details of individual research groups, industries and utilities. Therefore, the collaboration and joint research studies that leverage competences across research groups, industries, and utilities, are feasible even in case of confidentiality constraints.

The underlying concept of virtual interconnection of laboratories in real time is being introduced as "Geographically Distributed Real-Time Simulation (GD-RTS)". GD-RTS refers to a concept of partitioning a monolithic simulation model into subsystem models that are simulated concurrently on multiple DRTS units located at geographically dispersed facilities. Interconnection and data exchange between DRTS systems is typically realized via a shared communication network, such as the Internet. As illustrated in Figure 2, geographically dispersed HIL setups can be interconnected based on GD-RTS concept. Therefore, comprehensive real-world testing of interconnection and interoperability between novel technologies and existing power grid can be performed without having the diverse HIL setups located at the same facility.

Interface quantities exchanged among DRTS units are variables defining power, typically current and voltage. The prime requirement for an accurate GD-RTS is conservation of energy at the interface. Energy must be neither generated nor stored at the interfaces between simulated subsystems. If conservation of energy is violated, simulation stability and fidelity cannot be 
ensured and results of GD-RTS are not valid. Simulation fidelity is a measure of how close are results obtained in GD-RTS to the simulation results obtained based on a monolithic model. It highly depends on a partitioning point that is selected to partition the monolithic model for the purpose of distributed simulation. Empirical studies showed that substation transformers and HVDC lines are suitable for model partitioning. Furthermore, interconnection of laboratories via the Internet introduces an inevitable communication latency as well as other characteristics of a shared communication network such as jitter, packet loss and packet reordering. As a result, conservation of energy at the interfaces can be significantly violated. Co-simulation Interface Algorithms (IA) are responsible for compensation of the effect of communication medium simulation stability and fidelity [5].

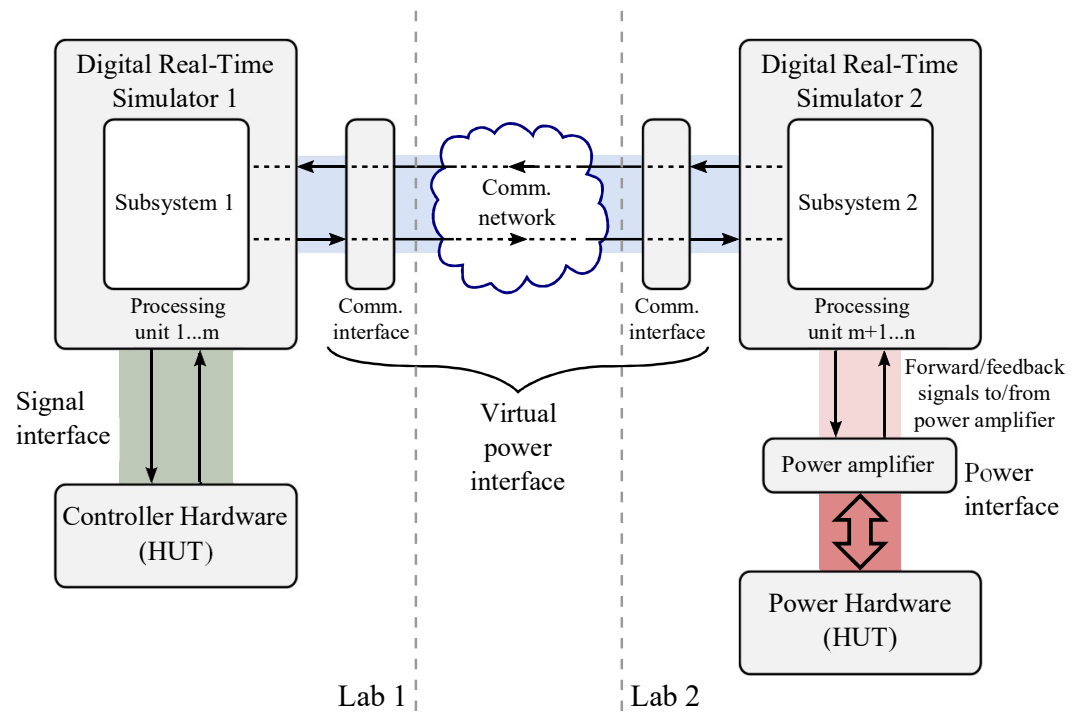

Fig. 2: Geographically Distributed Real-Time Simulation

\section{CO-SIMULATION PLATFORM}

Distributed simulation relies on a co-simulation framework for interfacing participating simulators with each other.

To support the vision of a global grid of simulators, a new co-simulation framework named VILLASframework has been developed by the RWTH Aachen University [7], [8]. Hardware-in-the-Loop simulations a demand models in the electromagnetic transients (EMT) domain which are executed in real time. With time-steps ranging from a 1 to $50 \mathrm{uS}$, this becomes a challenging task which handled by specially optimized real-time simulation targets. Scaling such simulations to thousands of nodes is currently impossible due to the computational limits imposed by a single target. VILLAS attempts to solve this limitation by coupling existing and proven digital real-time simulators into clusters through the Internet.

The framework consists of four components which can be used as building blocks for arbitrarily large co-simulation setups.

Its first component, VILLASnode, is a gateway for simulation data and measurements. It provides a variety of interfaces to existing real-time simulation targets as well as adapters to commonly used protocols like, UDP, MQTT, AMQP or IEC 61850. The main difference to other existing frameworks is its modular and decentralized architecture as it does not rely on a central broker and scheduler to pace the simulation. Instead simulators are interfaced to the VILLASnode gateways which in turn are connected to other gateways and simulators as shown in Figure 3. Similar to the architecture of the Internet, complex co-simulation scenarios composed by a collection of peerings without a central authority can be created.

Without a global scheduler, each simulator itself is responsible to synchronize its shared task execution to a global time reference. No synchronization between the targets itself is performed. Apart from handling the data exchange, the gateway is responsible for monitoring the interfaces, collecting results and statistics.

The second component, VILLASweb is a web interface enabling participant to remotely monitor the simulation via a standard web browser. Graphical dashboards allow the user to customize their view of the simulation according to their interests. Simulation results are streamed via WebSockets in near real-time to the browser and rendered by a variety of widgets like plots, gauges or text boxes. This live view of the simulation state is crucial for enabling interaction. Buttons, sliders and dial widgets permit the user to affect the course of the simulation. An example of such a web-based visualization is shown in Figure 4. 
Workstation \#1..n

Public Server
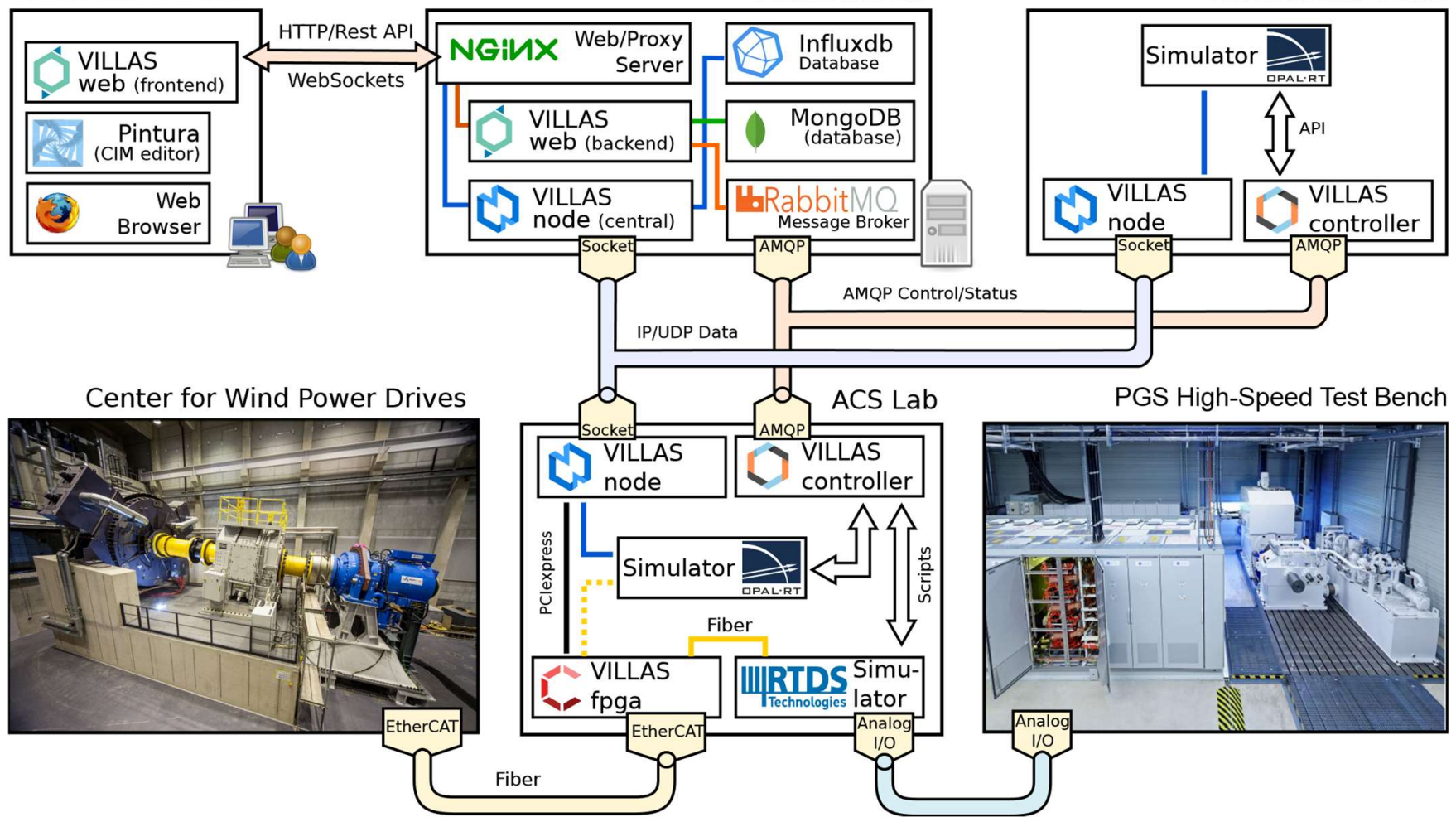

PGS High-Speed Test Bench

Fig. 3: Example of VILLASframework co-simulation platform.

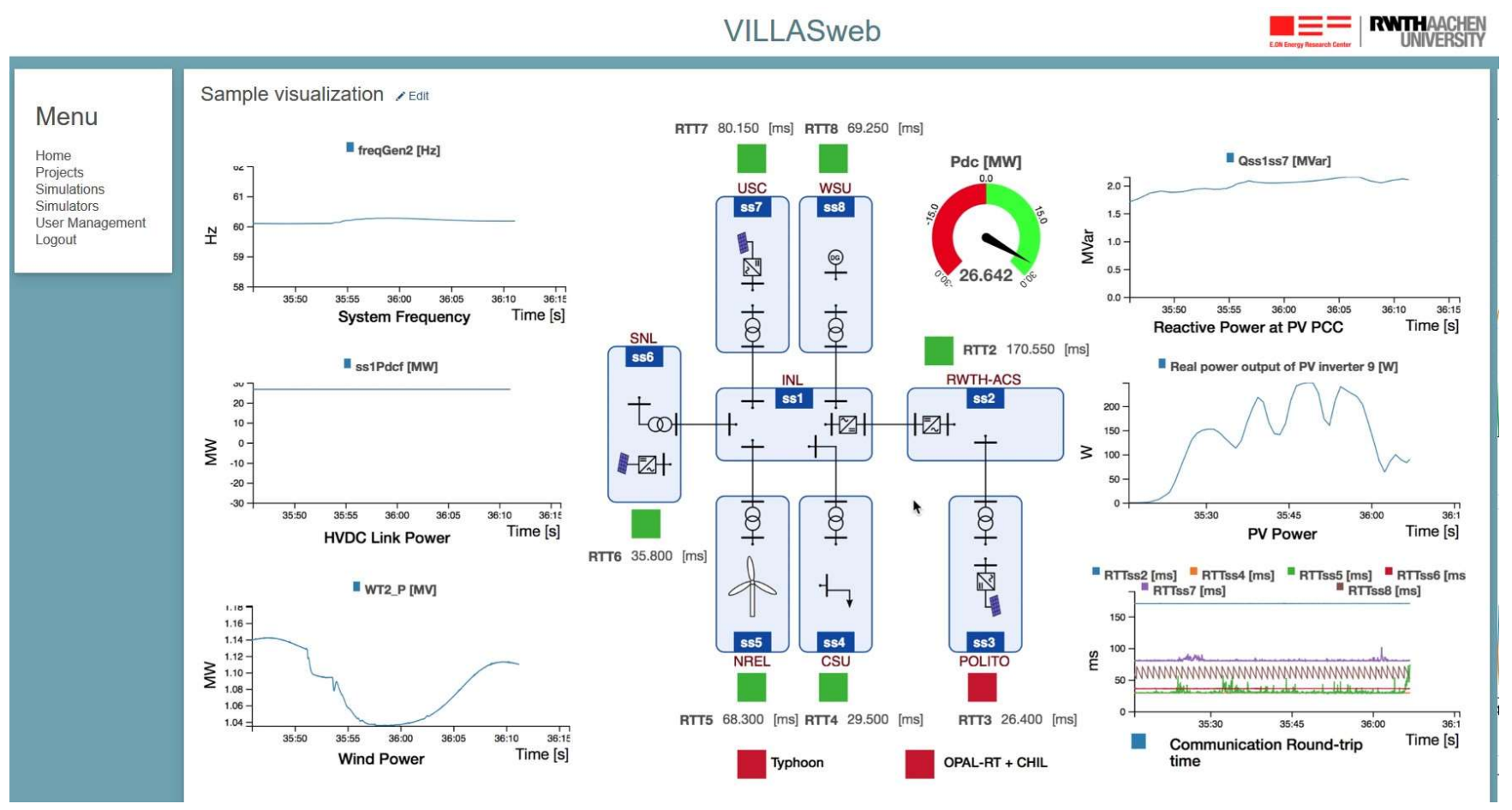

Fig. 4: Screenshot of web-based live visualization of simulation status and results. 


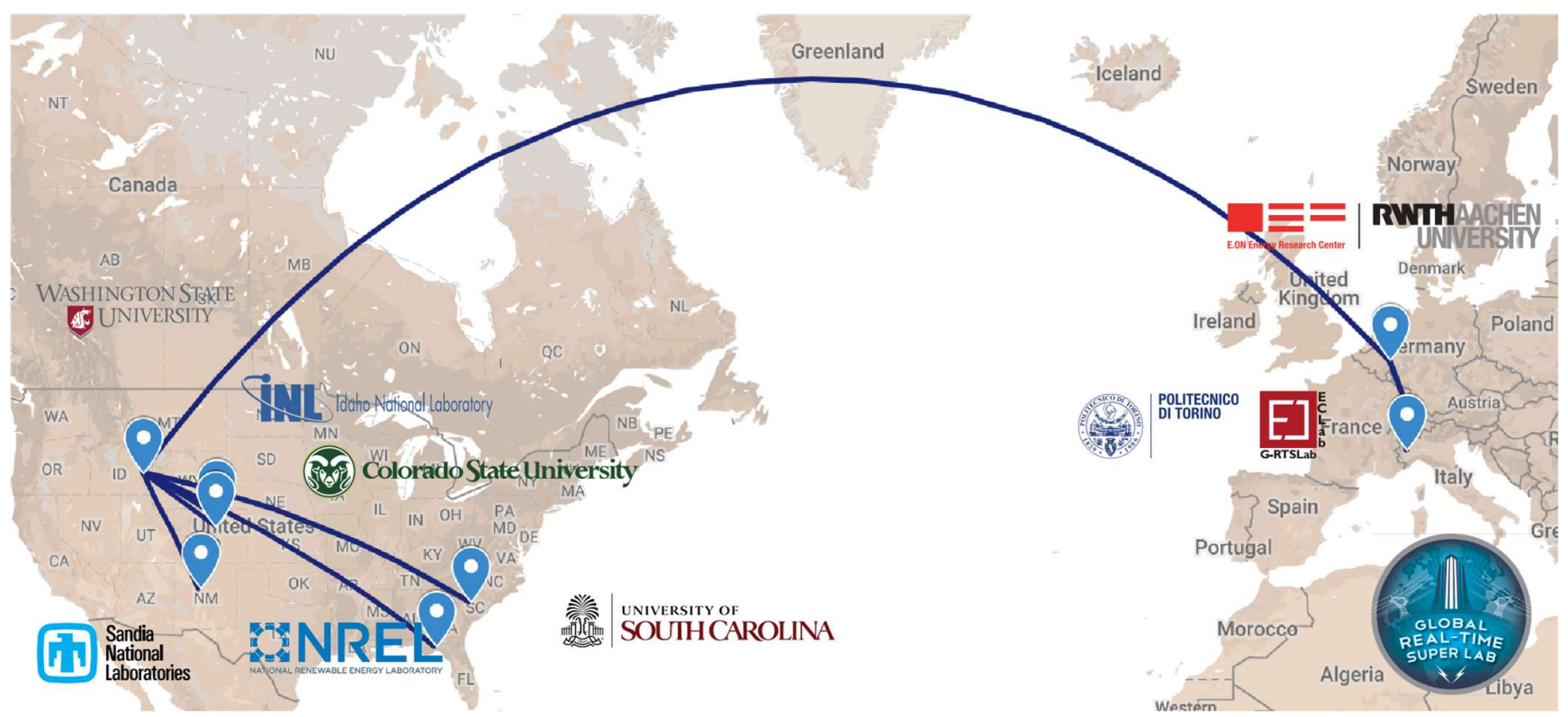

Fig. 5: RT-Super Lab participants and their interconnections.

\section{The Global Real-Time Super lab Demonstration}

RWTH Aachen University gained first insights and experience with virtual interconnection of laboratories in collaboration with SINTEF, a research organization with facilities in Norway. In 2014, RWTH and SINTEF demonstrated the feasibility of joint simulation based on long-distance interconnection of OPAL-RT systems located in Aachen, Germany and Trondheim, Norway. At the same time, following the vision of large-scale vendor-neutral virtual interconnection of laboratories, RWTH started the design and development of VILLASframework. In October 2015, RWTH, POLITO and two facilities of the European Commission's Joint Research Centre (JRC) demonstrated virtual interconnection of four laboratories in Europe and established European Realtime Integrated Co-simulation laboratory (ERIC-LAB). Earlier that year, two national laboratories in the USA, INL and NREL, successfully connected RTDS systems at their facilities. The work towards transatlantic interconnection of laboratories located in Europe and the USA had started in 2016 with collaboration of RWTH and INL. Another six research institutions joined this initiative in the following year with the main objective to establish the Global Real-Time Super Lab across Europe and USA.

In September 2017, the largest geographically distributed real-time simulation of a large power system was performed as the Global Real-time Super Lab Demonstration [9]. In total of eight laboratories utilizing ten digital real-time simulators from the three major vendors OPAL-RT, RTDS and Typhoon were interconnected over the Internet using the previously presented VILLASframework. Figure 5 shows the geographically location of the involved laboratories spanning the United States of America as well as Germany and Italy in Europe.

Table I and Figure 6 provide an overview of participants of Global RT-Super Lab demonstration and the outline of simulation models and laboratory setups. In the US, the Idaho National Laboratory (INL) took a central role by simulating the 9-bus Western Systems Coordinating Council (WSSC) transmission benchmark network on Real-Time Digital Simulator (RTDS) system. Likewise in Europe, the RWTH Aachen University contributed the RTDS-based simulation of a 12-bus European transmission network benchmark model proposed by CIGRE. The transatlantic link between INL and RWTH was established by a long distance HVCD line with both RWTH and INL laboratory simulating one converter station. The rationale behind this choice is the vision of Global Grid, a globally interconnected power grid based on Ultra HVDC lines connecting continents across the globe [10]. HVDC link allows for interconnection of grids with different system frequencies. Thus, laboratories in the USA and Europe can focus on simulation and testing of power grids with characteristics of the real local grids. Furthermore, selection of HVDC link as a partitioning point preserves simulation stability for large communication latency between RWTH and INL laboratories.

There were distribution networks connected to backbone transmission networks at NL and RWTH, including a Controller-HIL setup contributed by the University of South Carolina (USC) and PHIL setups of a wind-turbine and a PV inverter provided by National Renewable Energy Laboratory (NREL) and Sandia National Laboratories (SNL), respectively. In general, the model was partitioned into the US and EU grid that includes transmission and distribution networks with diverse PHIL and CHIL of devices that are representative of the novel technologies being interconnected. Politecnico di Torino (POLITO) utilized OPALRT system for simulation of CIGRE European distribution network benchmark model with PV units and interconnection to the transmission system simulated at RWTH. SNL contributed the 7-bus simplified distribution system simulated on OPAL-RT with PHIL setup for 
a physical PV inverter. PHIL setup of a wind-turbine together with a simplified 3-bus distribution system simulated on RTDS was contributed by NREL. The laboratory setup at USC included Controller-HIL with National Instruments CompactIO controllers, hardware Apposite N-91 for emulation of communication network and OPAL-RT system for simulation of modified IEEE 123-bus distribution test system. The controllers are responsible for controlling reactive power of PV inverters and aim at minimizing power losses. IEEE 13-bus distribution test feeder and interconnection to WSCC transmission model at INL was simulated on OPAL-RT system along with advanced load management systems at Colorado State University (CSU). Washington State University (WSU) utilized RTDS for simulation of a resilient 9-bus microgrid based on Consortium for Electric Reliability Technology Solutions (CERTS) concept. Typhoon system at INL was utilized for simulation of the reduced microgrid test feeder with active generation, representing a reference industry feeder known as a Banshee. Transformers are identified as suitable partitioning points between transmission and distribution systems with respect to simulation stability and fidelity. This choice also allowed each research group to simulate systems relevant for their competence and research focus. DRTS systems at all laboratories perform electromagnetic transient simulation with the time step of $50 \mu$ s, except for OPAL-RT simulator at USC utilizing $100 \mu \mathrm{s}$ for simulation of large-scale distribution system.

\begin{tabular}{|c|c|c|c|c|c|}
\hline Site & Grid & \# Busses & Peers & HIL & Simulator \\
\hline Idaho National Laboratory, USA & WSCC & 9 & $\begin{array}{l}\text { RWTH, WSU, } \\
\text { USC, SNL, } \\
\text { NREL, CSU }\end{array}$ & - & $\begin{array}{l}\text { RTDS, } \\
\text { Typho } \\
\text { on \& OPAL-RT }\end{array}$ \\
\hline RWTH Aachen University, Germany & CIGRE HV & 12 & INL, POLITO & - & RTDS \\
\hline Politecnico di Turin, Italy & CIGRE MV & 14 & RWTH & - & OPAL-RT \\
\hline Sandia National Laboratory, USA & Distribution grid & 7 & INL & $\begin{array}{l}\text { PHIL for PV } \\
\text { inverters }\end{array}$ & OPAL-RT \\
\hline University of South Carolina, USA & $\begin{array}{l}\text { IEEE distribution } \\
\text { test system }\end{array}$ & 123 & INL & $\begin{array}{l}\text { Controller } \\
\text { HIL, Network } \\
\text { Emulation }\end{array}$ & OPAL-RT \\
\hline Colorado State University, USA & $\begin{array}{l}\text { IEEE distribution } \\
\text { test feeder }\end{array}$ & 13 & INL & - & OPAL-RT \\
\hline
\end{tabular}

TABLE I: Simulation models of RT-Super Lab.

Each laboratory was hosting a dedicated instance of the VILLASnode simulation gateway to collect statistics and exchange interface quantities with the central gateway at INL, while POLITO was exchanging interface quantities with RWTH. As nodes with multiple co-simulation interfaces, INL and RWTH had to merge the streams from its peers and send them collectively to the local RTDS simulator. In addition, all laboratories were sending monitoring data to INL, which has been hosting the VILLASweb interface with the visualization shown in Figure 4. VILLASweb visualization was utilized by all participants to obtain a holistic view on the overall simulation and presented to the guests.

Between the gateways, the User Datagram Protocol (UDP) has been chosen for exchanging raw simulation data. Dedicated tests revealed an optimal packet sending rate of around 1000-2000 p/S which keep network congestion and packet loss to a minimum. For security reasons, all network traffic between the laboratories was encrypted with IPsec VPN.

The central role of INL has simplified the overall process to bring up the simulation. Testing procedure was divided in four main stages: pre-unit testing, unit testing, integration testing, and system testing. Pre-unit testing had an objective to verify local laboratory setups and data exchange between a DRTS system and VILLASnode. Second, simple connection and point-topoint unit tests have been performed between INL and its peers as well between RWTH and POLITO. A benchmark co-simulation model has been designed for unit tests to provide reference model with high simulation fidelity for the first GD-RTS tests. Following them, an integration test phase successively added peers to build up the full scenario. Finally, during system testing the simulation scenario and VILLASweb visualization were adjusted and tuned for the final Global RT-Super Lab demonstration. 


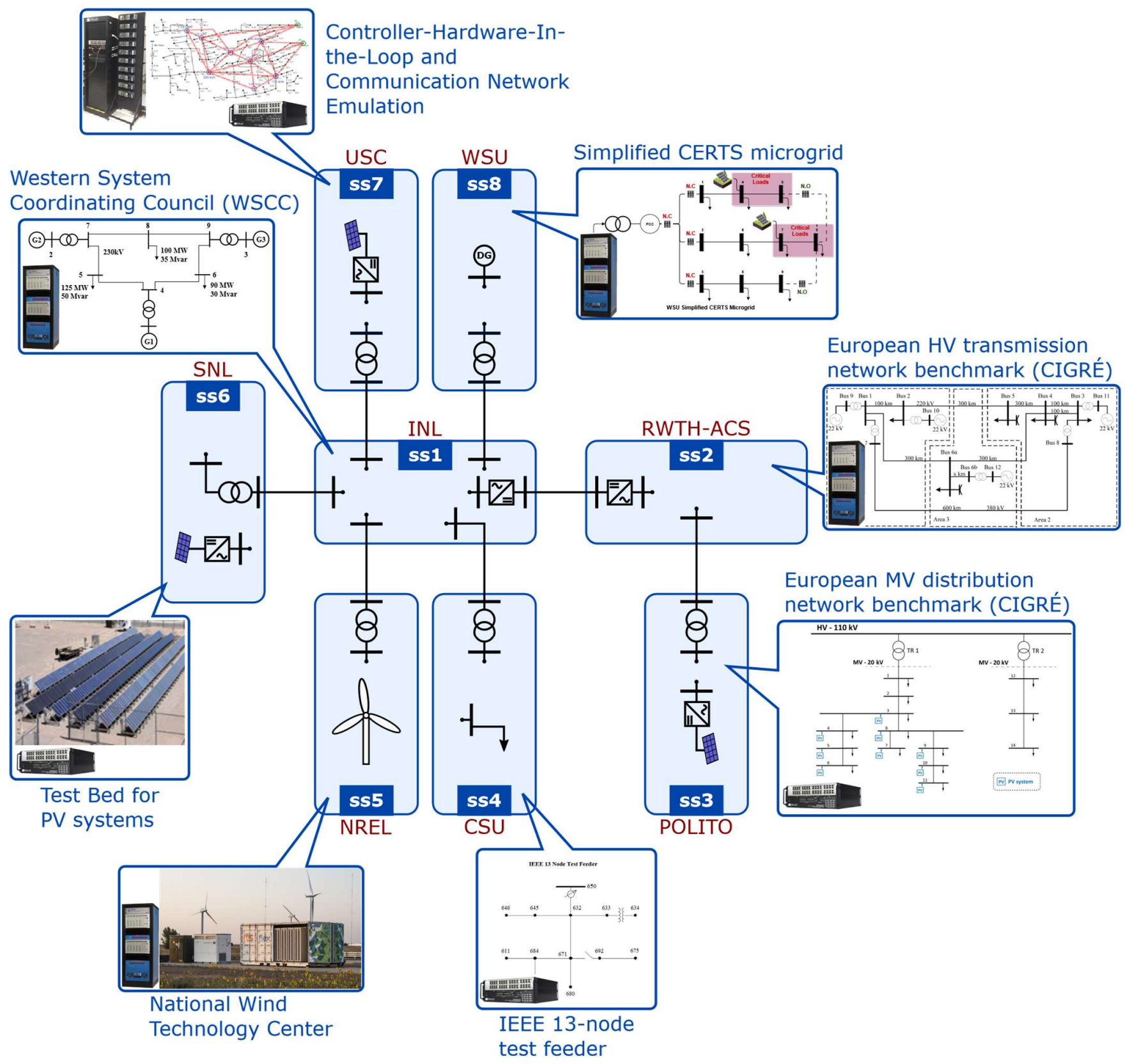

Fig. 6: RT-Super Lab Scenario

\section{A. Simulation Scenario and Results}

The simulation scenario was designed with the objective to leverage key resources and competence of each laboratory and demonstrate the advantage of a network of laboratories for studying next-generation power grids.

Following the demo start-up procedure and after the overall interconnected system has reached a steady-state operating point with respect to co-simulation interfaces, the ComapactRIO controllers at USC described in the previous section were activated. As a result, the reactive power at the Point of Common Coupling (PCC) of interconnection of the distribution system at USC to the WSCC transmission system at INL was reduced. Power measurements at the co-simulation interfaces at INL and USC are illustrated in the Figure 7 and indicate high level of simulation fidelity as well as the described scenario.

The transient across HVDC link between RWTH and INL was initiated by changing the set point for active power reference by $25 \mathrm{MW}$ of converter station at the transmission system at RWTH. This results in the decrease of power transfer from INL to RWTH across HVDC link.

As a result, system frequency of WSCC transmission system at INL increases due to net excess generation. 
Following the frequency increase, wind turbine at NREL decreases the active power output as the control of wind turbine includes droop curve for system support during frequency deviations. The total active power of NREL system at PCC with WSCC system at INL measured at INL and NREL terminals of the co-simulation interface is illustrated in the Figure 8.
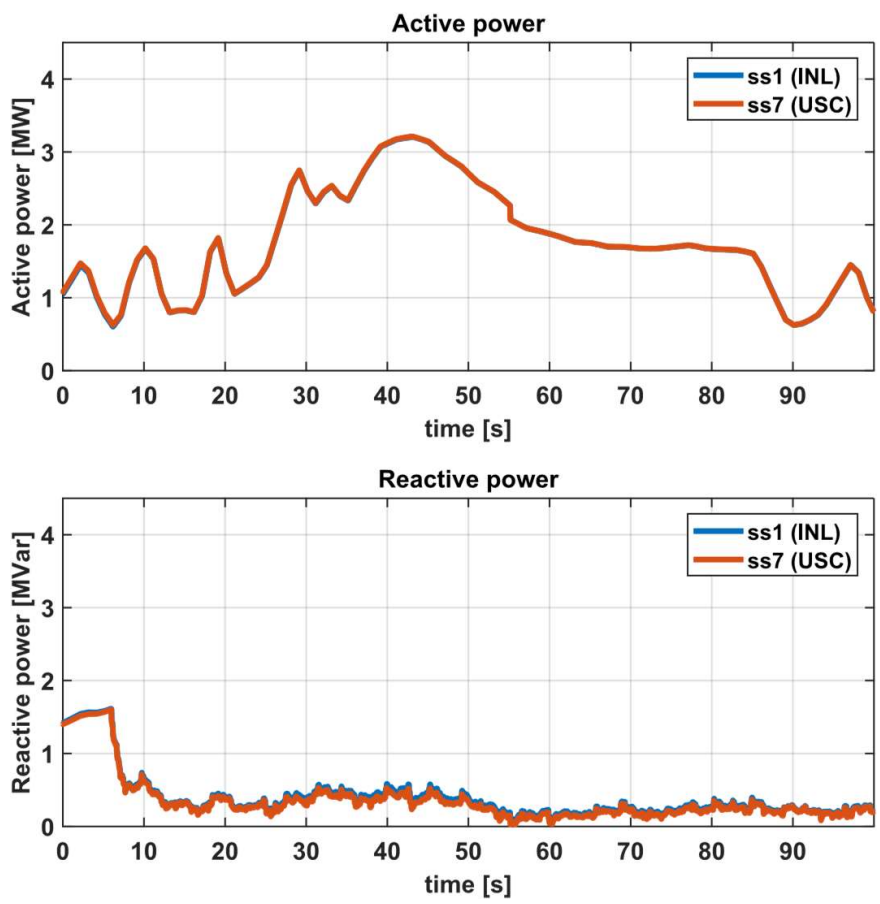

Fig. 7: Power measurements at INL-USC co-simulation interface following the activation of controllers at USC

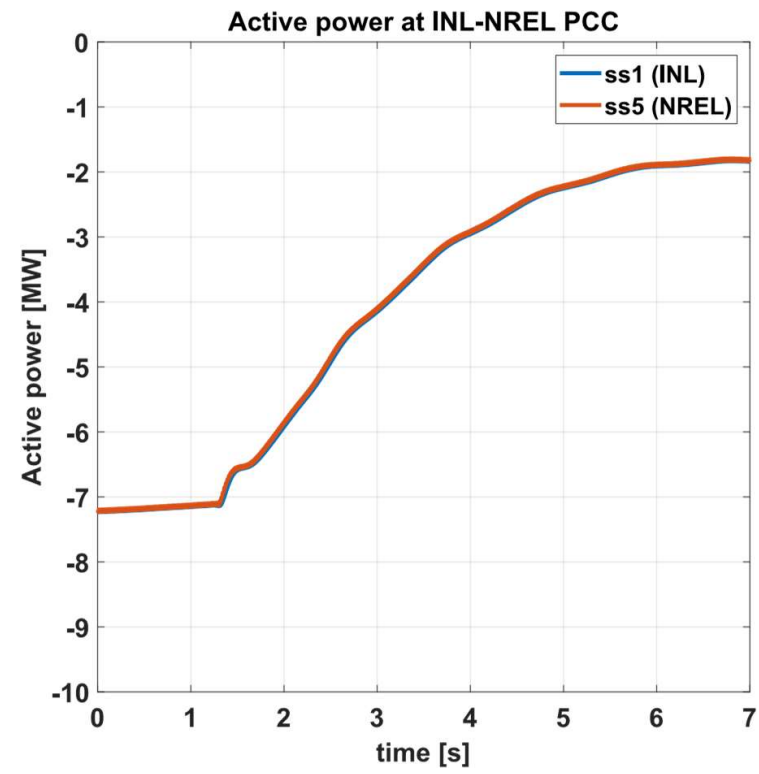

Fig. 8: Response of wind turbine at NREL following the frequency increase 


\section{CONCLUSION}

Global RT-Super Lab has been successfully established with its first large-scale transatlantic demonstration. VILLASframework significantly reduced the efforts by enabling modular and flexible interconnection and coordination of multi-vendor virtually interconnected distributed platform. Eight geographically distributed laboratories jointly performed a comprehensive simulation of a transatlantic HVDC interconnection between the benchmark transmission systems of the USA and European grids, including local transmission-distribution interactions and capability assessment of distributed generation units to support the grid operation. Global RT-Super Lab provides collaborative environment for such holistic studies by leveraging individual resources and competences in a flexible way.

\section{ACKNOWLEDGMENT}

The authors would like to thank Marcelo Masera as part of the European Commission for his initial ideas and support for a global power system simulation infrastructure. This work was supported by the RESERVE, which is an EU Horizon 2020 project funded under grant agreement No 72748. The authors would like to thank and acknowledge the financial support for projects related to interconnectivity between labs from the Department of Energy (Fuel Cell Technologies Office, Water Power Technologies Office) \& the Laboratory Directed Research and Development Office, INL.

\section{REFERENCES}

[1] T. Ackermann, T. Prevost, V. Vittal, A. J. Roscoe, J. Matevosyan, and N. Miller, "Paving the way: A future without inertia is closer than you think," IEEE Power and Energy Magazine, vol. 15, no. 6, pp. 61-69, Nov 2017.

[2] J. Rocabert, A. Luna, F. Blaabjerg, and P. Rodrguez, "Control of power converters in ac microgrids," IEEE Transactions on Power Electronics, vol. 27, no. 11, pp. 4734-4749, Nov 2012.

[3] J. A. Suul, S. D'Arco, and G. Guidi, "Virtual synchronous machine-based control of a single-phase bi-directional battery charger for providing vehicletogrid services," IEEE Transactions on Industry Applications, vol. 52, no. 4, pp. 3234-3244, July 2016

[4] D. Raisz, A. Musa, F. Ponci, and A. Monti, "Linear and uniform system dynamics of future converter-based power systems," in 2018 IEEE Power Energy Society General Meeting, August 2018, pp. 1-5.

[5] M. Stevic, A. Estebsari, S. Vogel, E. Pons, E. Bompard, M. Masera, and A. Monti, "Multi-site European framework for real-time co-simulation of power systems," IET Generation, Transmission Distribution, vol. 11, no. 17, pp. 4126-4135, 2017.

[6] M. D. O. Faruque, T. Strasser, G. Lauss, V. Jalili-Marandi, P. Forsyth, C. Dufour, V. Dinavahi, A. Monti, P. Kotsampopoulos, J. A. Martinez, K. Strunz, M. Saeedifard, X. Wang, D. Shearer, and M. Paolone, "Real-time simulation technologies for power systems design, testing, and analysis," IEEE Power and Energy Technology Systems Journal, vol. 2, no. 2, pp. 63-73, June 2015.

[7] S. Vogel, M. Mirz, L. Razik, and A. Monti, "An open solution for next-generation real-time power system simulation," in 2017 IEEE Conference on Energy Internet and Energy System Integration (EI2), Nov. 2017, pp. 1-6.

[8] Forderer der Energie- und Informationstechnik f" ur zuk" unftige Netze Aachen e.V., "FEIN Aachen e.V. - Software Projects." [Online] Available:" http://www.fein-aachen.org/projects/

[9] INL Media Relations, "Eight-lab simulator linkup aims to boost future electric grid stability," Jul. 2017. [Online]. Available: https: //www.inl.gov/article/eight-lab-simulator-linkup-aims-to-boost-future-electric-grid-stability/

[10] S. Chatzivasileiadis, D. Ernst, and G. Andersson, "Chapter 14 - Global power grids for harnessing world renewable energy," in Renewable Energy Integration, second edition ed., L. E. Jones, Ed. Boston: Academic Press, 2017, pp. $161-174$. 